International Journal of Biomedicine I June 2019 - Volume 9, Issue Suppl_1: Abstracts From the Second Russian International Conference "Cryo-electron microscopy 2019: achievements and prospects"

DOI: 10.21103/IJBM.9.Suppl_1.OR25

\title{
Abstract OR-25: Fitting Structural Fragments into Electron Microscopy Density Maps Using Spectral Shape Descriptors
}

\author{
Amihai Holzer ${ }^{1}$, Dina Schneidman-Duhovny ${ }^{1,2}$ \\ ${ }^{1}$ School of Computer Science and Engineering, The Hebrew University of Jerusalem, Jerusalem, Israel \\ ${ }^{2}$ Department of Biological Chemistry, Institute of Life Sciences, The Hebrew University of Jerusalem, \\ Jerusalem, Israel
}

Background: Macromolecular assemblies play a major role in cellular processes. Atomic resolution structural characterization of these machineries is necessary for understanding and modulating their function. The resolution revolution in cryo-EM paved a way for solving structures that for years resisted X-ray crystallography. This progress introduced a need for a new generation of computational methods for automatic structure determination with high and medium resolution cryo-EM density maps.

Methods: We present a novel method for rapid fitting of structural fragments into high and medium resolution density maps. The fragments can range in size from peptides to large proteins. The method utilizes spectral Heat Kernel Signature (HKS) shape descriptors, that are used in 3D shape recognition in Computer Vision. The fragment descriptors are fitted to the descriptors of the density map using the Geometric Hashing approach.

Results: We benchmark the method on a set of maps with resolved atomistic structures in the 3.5$6 \AA ̊$ resolution range (Fig.1).
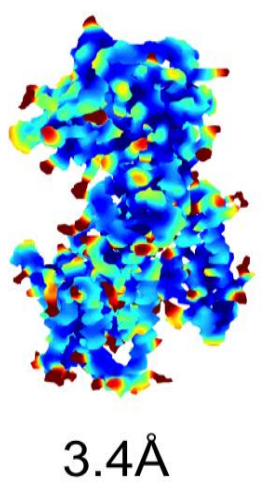

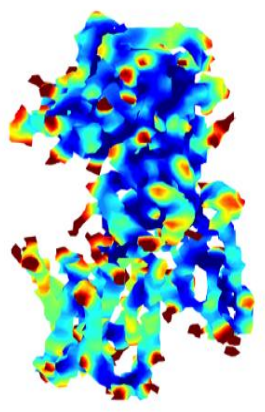

$4.5 \AA$

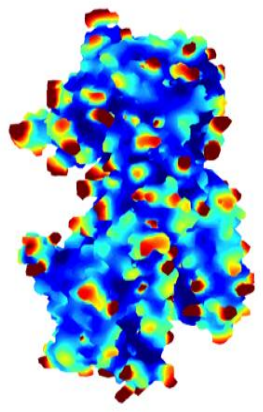

$4.0 \AA$

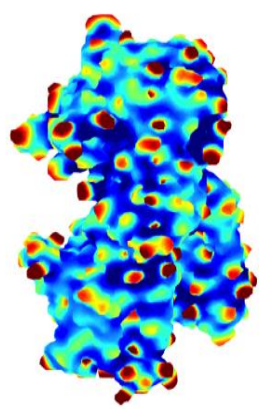

$6.0 \AA$

Fig. 1. HKS descriptors of experimental (resolutions 3.4 and 4.5A) and simulated maps (resolutions 4 and $6 \AA$ ) of $\gamma$-secretase. The coloring is according to the sum of HKS descriptors computed at 27 different time points.

Conclusion: The method is an important building block towards automated ab initio structure modeling. 
Key Words: atomic resolution structure modeling • EM density map fitting International Journal of Biomedicine. 2019;9 Suppl 1: S16. doi: 10.21103/IJBM.9.SuppI_1.OR25 C2019 International Medical Research and Development Corporation 